\title{
Programa work-flow para un servicio de consultas a medida
}

\author{
Por Tomàs Baiget
}

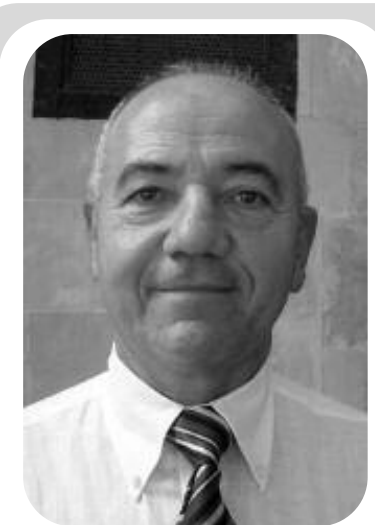

Tomàs Baiget trabaja en la Subdirección de Difusión Estadística del Instituto de Estadís tica de Catalunya (Idescat) como responsable de proyectos.

Resumen: Lo que más valoran los usuarios de los servicios de información en general es la rapidez en la respuesta, tanto si ésta resulta ser positiva como negativa. Se describen las características del Tramitador de Expedientes Digital (TED), un sistema de work-flow implementado en el Servicio de Atención de Demandas del Idescat, que permite gestionar electrónicamente todas las consultas de información estadística, con lo cual se minimizan considerablemente los tiempos de respuesta. Los usuarios rellenan un formulario electrónico en la web, reciben un acuse de recibo y, posteriormente, los resultados por correo electrónico. Con el TED las consultas circulan hasta uno o varios jefes de área (según la complejidad del tema), los cuales asignan uno o varios técnicos para elaborar las respuestas. Los ficheros y resultados finales los reúne la persona que haya sido designada en cada caso para «firmar» la salida (generalmente en función del tipo de solicitante). El sistema permite estandarizar los procedimientos, paso ineludible para poder obtener una certificación de calidad ISO.

Palabras clave: Servicio de atención al público / Tramitación de expedientes digital (TED) / Sistema electrónico / Work-flow / Calidad / Servicio integrado / Sistema corporativo

\section{Title: Workflow programme for a tailored enquiry service}

Abstract: One of the most valued aspects of information services in general is the immediacy of the answers, be these positive or negative. The Digital Dossier Transmitter is a work-flow system implemented by the Idescat's Enquiry Service that allows queries from citizens about statistical information to be managed electronically, resulting in considerably shortened response times. After submitting the electronic form available on the web, users are sent an acknowledgement message once their queries are validated and, subsequently, they receive the requested results by e-mail. Queries are circulated to one or more Area Heads (according to the complexity of the subject), who then assign them to the appropriate experts for drafting the answers. The final files and results are gathered together by persons designated, in each case, to «sign» the response (the designation is generally based on the type of requestor). The system allows procedures to be standardised, an unavoidable step for obtaining the ISO quality certification. Approximated implementation costs are given.

Keywords: Help desk service / Digital record processing / Electronic system / Work-flow / Quality / Integrated service / Corporate system

Baiget, Tomàs. «Programa work-flow para un servicio de consultas a medida». En: El profesional de la información, 2006, septiembre-octubre, v. 15, n. 5, pp. 364-372.

\section{Introducción}

El Instituto de Estadística de Cataluña (Idescat) es la oficina estadística oficial del gobierno autonómico de Cataluña (Generalitat de Catalunya). Tiene una plantilla de 120 y produce tanto estadísticas propias como en cooperación con el Instituto Nacional de Estadística (INE), así como explotaciones posteriores del censo de habitantes del $I N E$, de interés local.

Las estadísticas elaboradas se difunden por medio de publicaciones (por ejemplo el Anuario Estadístico de Cataluña, suministrado como libro, en pdf o en Excel, online y cd-rom), una biblioteca especializada en la sede del Instituto, de acceso libre y gratuito, y sobre todo, cada vez con mayor actividad, una web. La web es actualmente el gran escaparate de difusión.

Para complementar estos canales hace años que funciona también un servicio de atención de demandas o consultas a medida, al que acuden tanto las personas que necesitan ayuda porque no saben encontrar la información, como los estudiosos, universitarios, técnicos de las administraciones, etc., que necesitan grandes volúmenes de in- formación en diferentes formatos, o requieren hacer cruces de variables concretas, etc.

Es este Servicio de Atención de Demandas $(S A D)$ el que vamos a describir a continuación.

\section{Diseño e implementación}

Hasta el año 2003 muchas personas se dirigían a diferentes técnicos del Idescat por distintos medios (biblioteca, correo postal, fax, correo-e, teléfono...) para hacer esas peticiones de información. A todas se las atendía, pero -exceptuando la biblioteca- al cabo del año la direc- 
ción desconocía con certeza cuántas demandas se habían elaborado, quién contestaba, cuánto tiempo se había dedicado, y cómo lo había hecho. El asunto era algo preocupante porque los técnicos estadísticos tienen que dejar sus tareas de producción para realizar esas consultas, que pueden requerir desde una simple media hora hasta días de trabajo. Por otro lado, dado que a veces los conceptos y las tablas estadísticas tienen bastante complejidad, había que preguntarse hasta qué punto los técnicos tenían que ayudar a los usuarios en sus análisis de los datos.

Un directivo calificó la situación de esa época como de "anarquía organizada". Aparentemente funcionaba bien, pero nadie sabía cómo.

Con el fin de llevar un control de esas peticiones, se encargó a la empresa STE Consulting ${ }^{1}$ que adaptara el software de work-flow Tramitador de Expedientes Digital (TED), que ya era propiedad de la Generalitat porque una consejería lo había comprado, para gestionarlas.

El proceso de acordar las especificaciones funcionales iniciales entre los técnicos del Idescat y de $S T E$, y diseñar la aplicación para las necesidades del $S A D$ fue exactamente "de libro", con el fallo típico que cuentan todos los manuales de sistemas de información: sobredimensionamiento de opciones y complicación innecesaria:

-Circuitos que luego no se usan nunca. Por ejemplo, el envío al letrado del Instituto de demandas para que dictaminara si con el suministro de los datos solicitados se podía infringir el secreto estadístico. Cuando raramente se da esta situación es mucho más fácil discutirlo personalmente, pues además hay casos y casos.

O la revisión de las respuestas de los técnicos por parte de los jefes, lo que originaba tediosas idas y

\section{Diagrama de flujo de SAD-TED}

Final $1=$ Otros mensajes (todo excepto consultas estadísticas)

Final 2 = Afloramiento de consultas ya contestadas previamente

Final 3 = Contesta la consulta el mismo servicio $S A D$

Final 4 = Contesta la consulta el responsable de la Unidad

Final 5 = Terminación forzosa y/o resolución por teléfono

Final 6 = Resuelve la consulta un técnico y contesta la Unidad

Final 7 = Envía la consulta el $S A D$, bien porque es comparti-

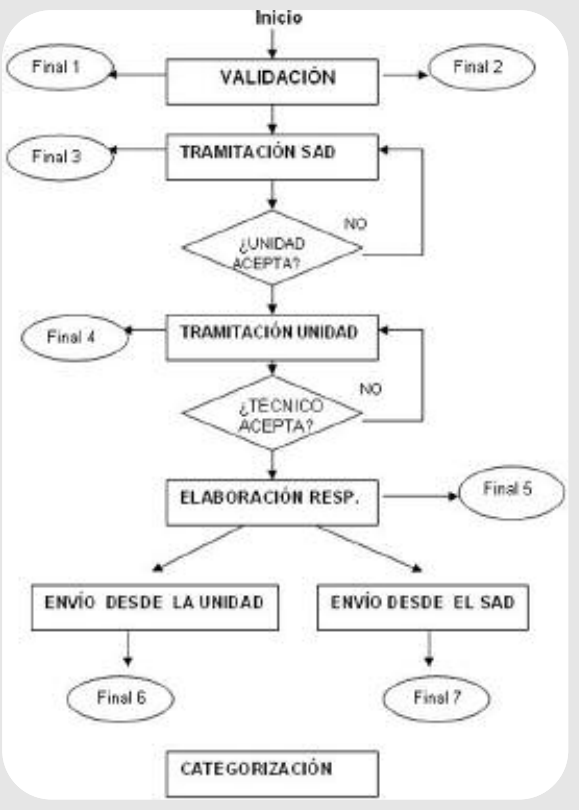
da con otra unidad o bien porque se tiene que preparar una carta de respuesta para el Director

Después de cada "final", excepto el primero, el sistema remite a la categorización o clasificación de la consulta.

venidas de los expedientes a través del sistema. Aunque se puede localizar perfectamente, la gente se liaba: "Y ahora ¿en la bandeja de quién está el expediente?".

O la introducción de observaciones por parte de la Dirección mientras dura la tramitación.

O el reenvío del procedimiento al Área de Asistencia Técnica Estadística para firmar un convenio de colaboración institucional cuando la petición es muy voluminosa.

O cuando el solicitante pide los datos certificados en papel, con firma y sello del registro oficial $\left(n^{\circ}\right.$ viviendas en pueblos para poder abrir una nueva farmacia, últimas residencias conocidas de personas desde los juzgados, existencia de determinados nombres de pila que determinado juez se niega a aceptar, etc.).

-Normalización de respuestas casi imposible ante la gran variedad de preguntas (según el tema, según el territorio, según la periodicidad, la serie temporal, según quien pregunta, estadística elaborada en Idescat y suministrada, estadística disponible próximamente, estadística inexistente, respuesta orientativa, explicación de cómo encontrar los datos en la web del Idescat o del $I N E$, respuesta sólo parcial, etc.). Después de que un técnico de STE redactara un montón de textos, lo único aprovechable fue poco más que el "Estimado señor/a. En respuesta a su petición del pasado día dd de mmmm pasado" y el "Le saluda atentamente". El dd y el mmmm correspondientes también los pone el programa automáticamente, pero todo es editable por la persona que redacta el mensaje.

-Etc.

La implantación del TED en la institución fue también "de manual": se contó con el apoyo del Director, muy interesado en el asunto, 
que reunió al personal para presentarlo. De no haberse hecho esta sesión, muy probablemente la aplicación no hubiera sido aceptada. Sin embargo, el relevo del Director -un cargo político- coincidiendo con la puesta en marcha del nuevo sistema ocasionó algún peligro de fracaso: un mando intermedio tuvo agallas para prohibir a sus técnicos usar el sistema: todas las consultas las tramitaban manualmente él y su secretaria (que era la única persona de aquella Área con acceso al TED) y los técnicos, pero con los expedientes impresos sobre papel (!). Pasados unos meses, y visto que no "peligraba su reino" esa área se integró al sistema como las demás.

Para seguir fieles a los manuales, que hablan del rechazo a los cambios, hubo otras cuantas oposiciones y críticas. Las dos personas encargadas del servicio y de resolver dudas de la gente tuvimos que aguantar frases del estilo como "¿Quién ha diseñado esta porquería de programa?", "Es absurdo, no tiene ni pies ni cabeza", «Eso no es un programa, es un jolgorio» (un inciso: creo personalmente que «jolgorio» está mal aplicado en este caso, pero es como lo dijo una empleada), etc. La situación la fuimos encauzando poco a poco con mucha paciencia, cara sonriente y acudiendo al instante cada vez que un técnico se atascaba en algún paso de la tramitación. Una gran suerte fue contar con unas fantásticas secretarias de Área que aprendieron rápidamente el funcionamiento y ayudaron en la tramitación a sus compañeros técnicos estadísticos.

\section{Nueva versión}

Todas las reuniones previas que se puedan hacer para definir las especificaciones técnicas y funcionales de un sistema de información resultan ser pocas. Siempre quedan cabos sueltos (¡esto también lo dicen los manuales!). En nuestro caso tuvimos dos sorpresas desagrada-

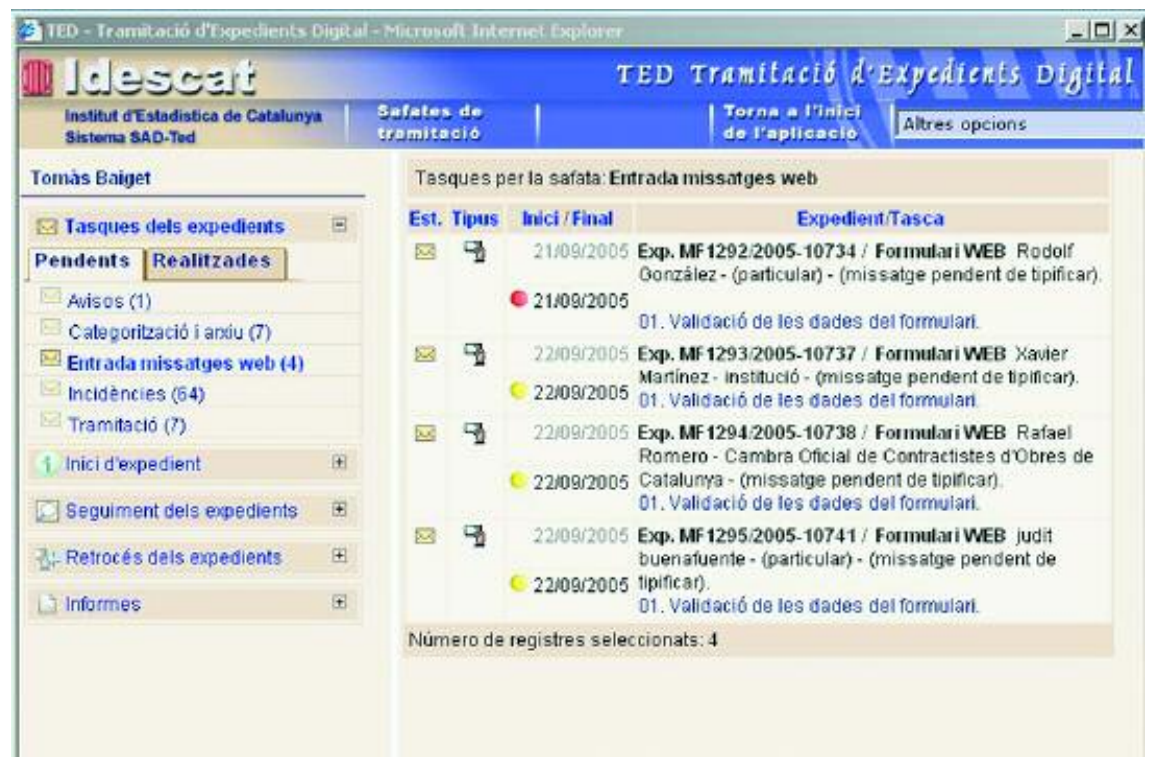

Figura 1. Ejemplo de pantalla de trabajo correspondiente al administrador del servicio. A la izquierda se ven las pestañas de tareas Pendientes y de tareas Realizadas lésta sólo se utiliza para comprobaciones, así como para recuperar una tarea hecha erróneamente si todavía nadie más ha intervenido a continuación) -el sistema tiene otros medios para retroceder los expedientes hasta el principio, si es necesario-.

Entre las tareas pendientes pueden observarse varias bandejas:

Avisos: Desde un área o unidad han rechazado una demanda por considerar que no la pueden o no la quieren realizar, habiendo escrito en el expediente una explicación o instrucciones para el $S A D$.

Categorización y archivo: Demandas terminadas, sólo pendientes de clasificar.

Entrada de mensajes web: En esta captura de pantalla aparece esta bandeja abierta y pueden verse los 4 formularios que contiene, pendientes de tipificar o de validar como demandas. Uno de ellos llegó hace dos días, y el sistema le pone un semáforo en rojo. Los otros tres llegaron ayer y tienen un semáforo amarillo. Los llegados en el día tienen un semáforo verde.

Incidencias: Aquí se pueden ver los 64 expedientes que en ese momento se estaban tramitando en total en el Instituto. El nombre de esta bandeja es un comodín para agrupar varias acciones que pueden realizarse con las consultas: rectificar datos del solicitante o de su petición (después de haberla aclarado por teléfono, p. ej.), terminar el expediente de forma forzosa (esto sólo el $S A D$ está autorizado para hacerlo) por cancelación de la consulta a petición del cliente, etc.

Tramitación: La «tramitación» para el SAD significa asignar las consultas a las distintas unidades del Instituto, o autoasignársela para responderla. En este último caso aparecerá en esta misma vista pero dentro de otra bandeja llamada Respuestas: Siguiendo en la parte izquierda de la pantalla, se ve el apartado Inicio de expediente, para rellenar manualmente el formulario de una nueva demanda; Seguimiento de expedientes, para consultar en cualquier momento en qué situación se encuentra una demanda, y quién(es) la tiene(n) pendiente en sus bandejas de trabajo; Retroceso de expedientes, y generación de Informes estadísticos.

bles, pues habíamos dado por sentado que tratándose de una aplicación sustentada en Oracle no habría ningún problema para combinar datos de la tramitación y obtener infinitas estadísticas del servicio.

También supusimos que recuperar registros de expedientes finalizados, de manera que el TED fuera como una base de datos de "know-how", era algo fuera de toda duda. Pues no:

—El módulo de obtención de estadísticas era muy pobre y no se acomodaba mínimamente a nuestras necesidades.

- Los expedientes sólo podían recuperarse por número, fecha $\mathrm{y}$ nombre del solicitante.

"Descubrir" esto representó una fuerte decepción. ¿Reclamar? Difícil, pues eso se tenía que haber especificado al principio. Por otro lado hay que reconocer que el personal de STE se volcó en el TED del Idescat e invirtió en él muchas más horas de las previstas. Al final se llega a dos conclusiones: 


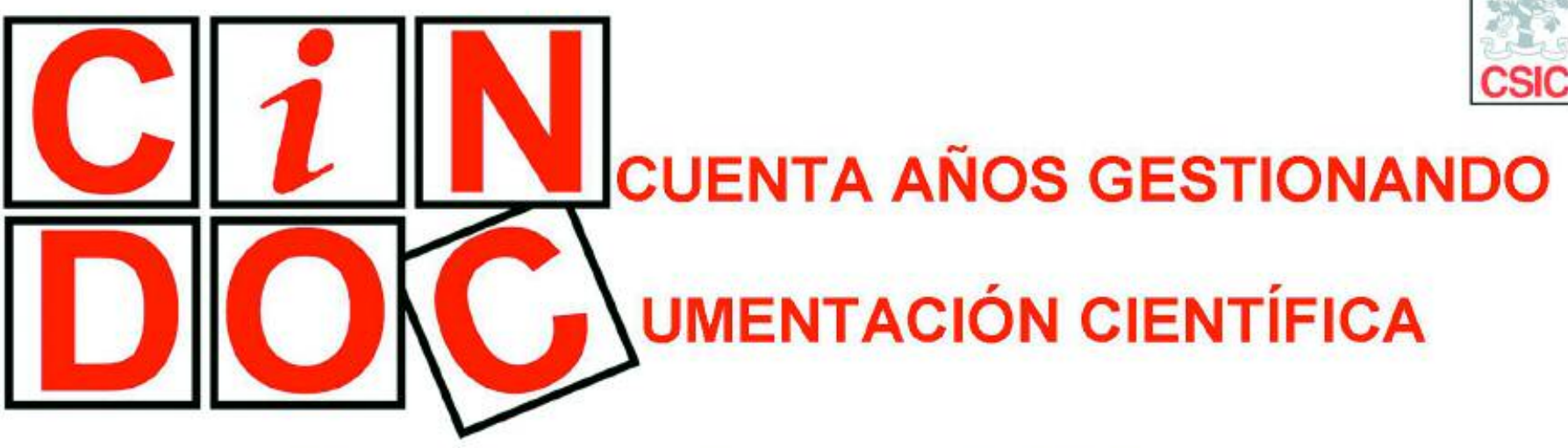

\section{Bases de Datos CSIC: Lo nuestro son los contenidos}

EI CSIC produce desde 1971 tres bases de datos bibliográficas con referencias de los artículos publicados en revistas científicas españolas y en otros documentos científico-técnicos
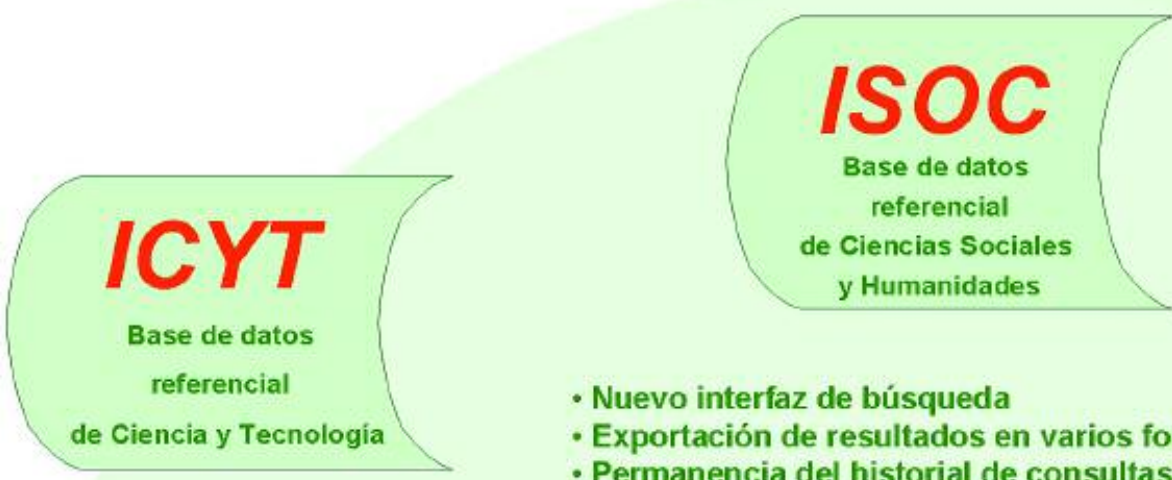

- Nuevo interfaz de búsqueda

- Exportación de resultados en varios formatos

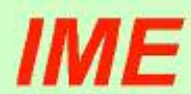

Permanencia del historial de consultas

- Disponibilidad de algunos documentos a texto completo

- Acceso gratuito a Sumarios

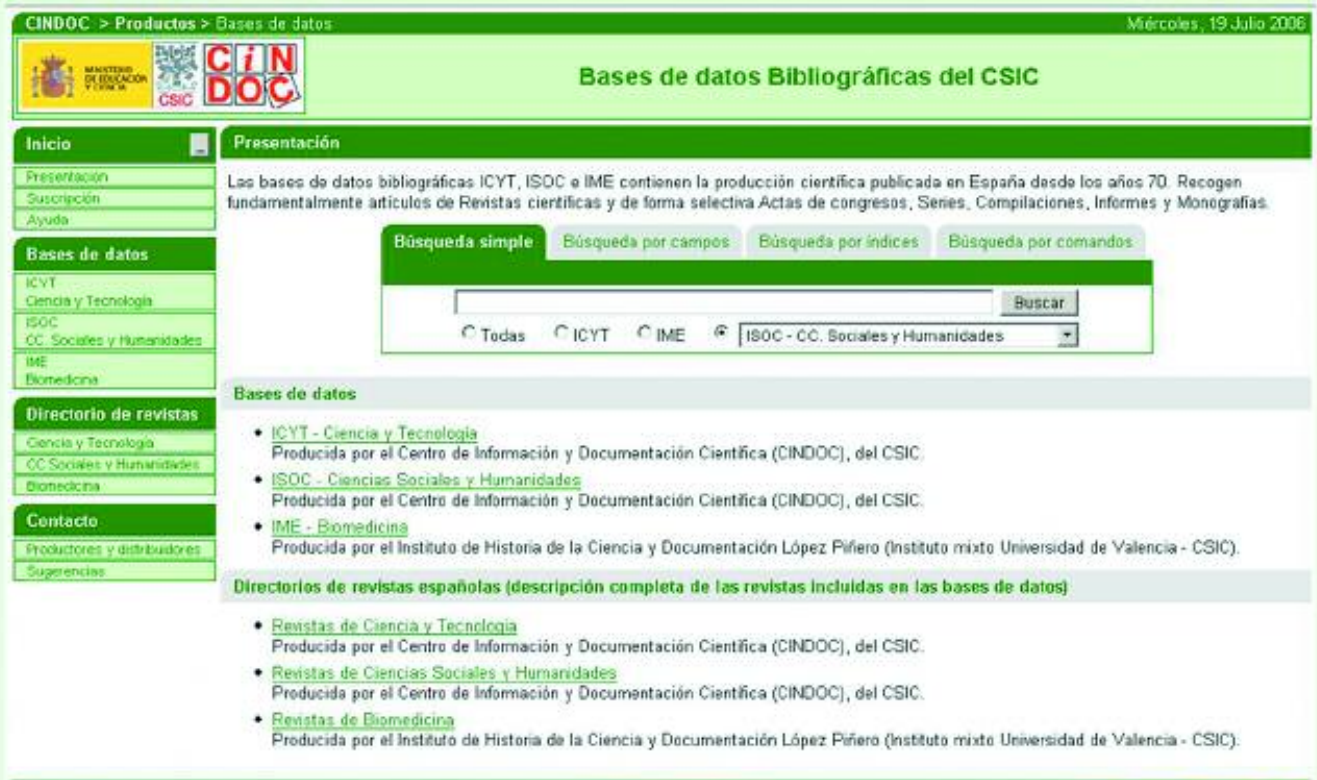

Copyright o CSIC. 2006

WSC

Consulte condiciones de suscripción en nuestro web

http://www.cindoc.csic.es 
1. Los sistemas de información siempre resultan ser más complicados de lo previsto, y es muy fácil pillarse los dedos después de haber dado un presupuesto.

2. El diseñador y el cliente (éste buen conocedor de las necesidades y de la idiosincrasia de la institución, y con poder para tomar decisiones) deben trabajar conjuntamente, sin dejar absolutamente nada expuesto a la "lógica" del otro. Hay demasiadas "lógicas". Así, por ejemplo, a nivel general, no es lo mismo diseñar un sistema de work-flow para miles de expedientes todos prácticamente iguales, que para cientos todos muy distintos. Y a nivel de detalle es inimaginable la cantidad de opciones «obvias» que resultan no serlo.

En el caso que describimos coincidió un interlocutor del Idescat muy exigente y meticuloso -grandes cualidades pero no para

\section{Temas de las consultas}

La información demográfica $\left(\mathrm{n}^{\circ}\right.$ de habitantes, inmigrados, movilidad, etc.) por municipios y comarcas es con diferencia la más solicitada al Servicio de Atención de Demandas del Idescat. Le sigue en interés el mercado de trabajo.

\begin{tabular}{|l|c|}
\hline \multicolumn{1}{|c|}{ Tema } & \begin{tabular}{c} 
No de $^{\circ}$ consultas \\
\hline $\begin{array}{l}\text { Población o demografía } \\
\text { (migraciones, etc.) }\end{array}$
\end{tabular} \\
\hline $\begin{array}{l}\text { Mercado de trabajo } \\
\text { (actividad, paro, etc.) }\end{array}$ & 187 \\
\hline Industria y energía & 96 \\
\hline Construcción & 84 \\
\hline $\begin{array}{l}\text { Nivel, calidad y } \\
\text { condiciones de vida }\end{array}$ & 71 \\
\hline $\begin{array}{l}\text { Comercio (incl. import- } \\
\text { export) y otros servicios }\end{array}$ & 65 \\
\hline $\begin{array}{l}\text { Macromagnitudes y } \\
\text { cuentas económicas }\end{array}$ & 63 \\
\hline $\begin{array}{l}\text { Agricultura, ganadería y } \\
\text { pesca }\end{array}$ & 58 \\
\hline Turismo & 58 \\
\hline $\begin{array}{l}\text { Onomástica (nombres y } \\
\text { apellidos) }\end{array}$ & 49 \\
\hline Salud, sanidad & 42 \\
\hline Educación, enseñanza & 41 \\
\hline Cultura y ocio & 36 \\
\hline Hogares y familias & 30 \\
\hline $\begin{array}{l}\text { Otros (medio ambiente, } \\
\text { finanzas, elecciones...) }\end{array}$ & 416 \\
\hline
\end{tabular}

diseñar sistemas de información-, que además frecuentemente complica las cosas sin necesidad, y un informático diseñador de STE muy ufano de su programa, que además tenía muy buena predisposición para demostrar que con el mismo es posible hacer todo tipo de trámite $\mathrm{u}$ opción que se insinuara. Si una tramitación permite un gran número de variantes, ello se traduce luego en la práctica en la necesidad de un gran número de clicks.

Después de funcionar durante 2004, y ya con la experiencia tanto del equipo de unas 10 personas del Idescat que más lo usan a diario, como de los técnicos de STE, se pidió a esta consultora una nueva versión simplificada y más ágil, que ahora funciona a plena satisfacción.

Hubo que pagar a STE el diseño de un nuevo módulo de obtención de estadísticas y, a falta de implementar otro módulo adicional (también de pago, por supuesto) para poder recuperar los expedientes por cualquier palabra, en la versión 2 existe un campo buscable de 30 caracteres (solamente) donde los técnicos pueden anotar algún descriptor o código. Algo es algo.

\section{Descripción del SAD-TED}

El sistema prevé la amplia variedad de solicitantes (desde estudiantes de bachillerato hasta técnicos de las administraciones públicas, empresas y consultoras) y de temas que puede haber, a diferente profundidad y extensión, de manera que la tramitación es lo suficientemente flexible para la gran mayoría de casos.

El Servicio está atendido normalmente por 2 personas a dedicación parcial. El número de usuarios inscritos (personal del Idescat) es de 93, y, como siempre, se cumple la ley de Lorenz o Pareto: el $20 \%$ realiza el $80 \%$ de las aproximadamente 1.650 demandas (2005).
Desde dentro del Idescat el personal accede al sistema a través de un navegador estándar iExplorer o FireFox.

\section{Inicio}

Las peticiones pueden entrar en el sistema de forma automática a través del formulario de la web que rellena el solicitante, o iniciando el expediente a mano cualquier persona del Idescat cuando las peticiones llegan por correo electrónico -a cualquier dirección personal-, correo postal, fax o visita.

Durante el primer año de funcionamiento se estuvieron contestando unas 540 demandas llegadas todavía en mensajes de correo electrónico dirigidas al buzón general del instituto idescat@idescat.net con otro mensaje rogando al remitente que rellenara el formulario de la web. Se observó que tener que rellenarlo fue disuasorio para un $8 \%$ de los solicitantes por correo.

En la nueva web -puesta en marcha en marzo de 2005- la citada dirección del Idescat no aparece en ningún sitio, por lo que en la actualidad ya prácticamente no llega ninguna petición a través de la misma.

Los formularios recibidos no inician expediente hasta que son validados por el encargado del $S A D$ como «Consulta estadística». Las otras posibilidades del menú desplegable son: «Comentario o sugerencia», «Asunto institucional», «Petición al Servicio de Prensa», «Agradecimientos» (perdón por decirlo, pero la gente se queda pasmada con nuestra rapidez), «Otros mensajes» $\mathrm{y}$ «Mensaje desestimable» (Final 1) cuando alguien ha enviado errores, pruebas, etc. Puede observarse, pues, que el $S A D$ actúa también como receptor de otros asuntos, contestando o redireccionando convenientemente, y ello es así porque el formulario es la única dirección electrónica publicada en 
la web para dirigirse al Instituto (al hacer click en «Consúltanos»).

Los solicitantes pueden adjuntar documentos en Word o Excel al formulario de la web (modelos de tablas solicitadas anteriormente para las que se pide la actualización, listas de municipios... y otros, como por ej. boletines de instituciones, CVs).

Siempre llega un pequeño porcentaje de peticiones «protocolarias» dirigidas al Director por fax o correo postal (éstas sufren el retraso de pasar por el Registro Oficial). En estos casos se introducen los datos a mano desde el formulario interno del SAD-TED (figura 1, «Inicio de expediente») y se adjunta el original escaneado al expediente.

Algunas veces las demandas se introducen a posteriori, sólo para hacerlas constar en el sistema (Final 2), pues alguien ha servido ya los resultados a los usuarios por un motivo urgente o por visita. Es el caso típico también de la Biblioteca, que a efectos estadísticos introduce en el sistema sólo la porción de demandas realizadas más complejas, -así como las que su personal no puede resolver en la sala-. Como es de suponer, la Biblioteca atiende otras muchas peticiones rápidas que se contabilizan aparte con muchos menos datos, pues no compensaría rellenar un formulario tipo $S A D-T E D$ para cada una de ellas.

\section{Tramitación}

Después de validar el formulario, el encargado del Servicio de Atención de Demandas (figura 1) puede optar por responder él mismo (por tratarse de una información que está en la web, para dar una respuesta orientativa hacia otro organismo, etc.) (Final 3) o bien mandarla a una o más unidades o áreas de trabajo ${ }^{1}$ del Idescat (producción de estadísticas demográficas, sociales, económicas, área de coyuntura, área de municipios y comarcas, pla-

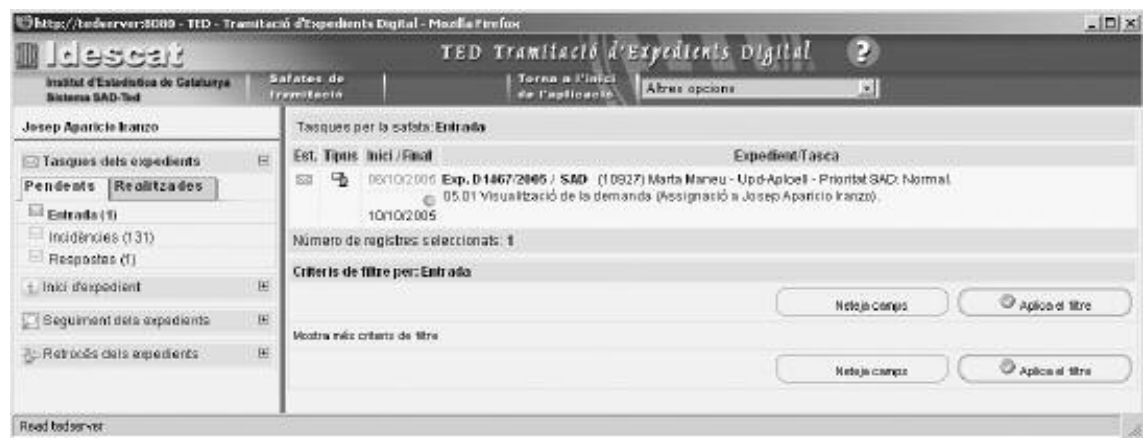

Figura 2. Aspecto de la pantalla de un técnico

En la bandeja de entrada tiene una demanda pendiente de visualizar. Si no lo hace hoy, mañana el semáforo verde se pondrá amarillo y al día siguiente la demanda rebotará a la bandeja del jefe de Área que se la ha asignado, con un aviso de "Técnico ausente».

Este mismo técnico tiene una demanda ya en la bandeja de Respuestas en espera de que termine de elaborar los resultados (por ej., unas tablas en Excel). El técnico redactará el mensaje de respuesta, adjuntará las tablas y finalizará su parte, pero si opcionalmente se había anotado que el expediente tenía que ser enviado por el jefe de área, el expediente irá a parar a la bandeja de éste (o de su secretaria) para ser revisado y enviado.

nes y métodos estadísticos, biblioteca, etc.).

Si la manda a más de una Unidad se origina una «demanda compartida» o «con subexpedientes». La única diferencia es que cuando estén listas todas las respuestas será el $S A D$ quien firmará el mensaje y efectuará el envío conjunto al solicitante (Final 7).

Para cada expediente el $S A D$ decide a priori quién tiene que firmar la respuesta, cosa que señala en un desplegable donde figuran: «Director», «SAD», «Responsable de Área» y «Técnico». Esta decisión puede ser cambiada desde cada nivel jerárquico hacia abajo. Hay que distinguir entre esta «firma»-que es más bien el lugar dónde irá a terminar el expediente- $y$ la firma real que luego se haga constar en el mensaje que recibe el solicitante.

$\mathrm{Si}$ una de las unidades retrasa mucho la respuesta (evidentemente, el personal encargado del Servicio va controlando las consultas pendientes) se puede enviar sólo la parte de los resultados ya lista, quedando el resto pendiente de envío. Cuando la unidad correspondiente finalice la respuesta, el sistema avisa al $S A D$ que tiene una respuesta preparada para enviar.
Cuando en el proceso normal se envía el expediente a una Unidad, el responsable de ésta puede juzgar que la petición no le corresponde y rechazarla, anotando las razones. El expediente reaparece en las bandejas de los encargados del Servicio, los cuales pueden reasignarlas a una unidad alternativa o intentar encontrar la información por sí mismos (haciendo llamadas, buscando en la web del INE, en Google, etc.). Siempre se intenta dar alguna información, o al menos una respuesta amable, pues para muchos ciudadanos resulta difícil saber dar con el sitio adecuado donde preguntar, a pesar de los teléfonos generales 010 y 012 .

Cuando el responsable de una Unidad acepta una demanda, tiene dos posibilidades: asignársela a sí mismo (Final 4) o mandarla a uno o varios técnicos (figura 2).

Una vez finalizada la respuesta por parte de un técnico, si éste está autorizado el sistema le permite enviar el mensaje con los resultados directamente al solicitante. En el cuerpo del mensaje (o en una carta de presentación adjunta, en word o pdf) puede firmar él mismo o la persona designada (Final 6).

En algunas ocasiones especiales en la Unidad pueden juzgar que es más eficiente y claro dirigirse al 
solicitante por teléfono: para explicar métodos, tablas o datos enviados anteriormente, por qué no se puede entregar determinada información, etc. (Final 5). Los ciudadanos acostumbran a agradecer enormemente este tipo de respuesta.

También puede suceder que una vez aclarada la demanda con el interesado se decida no continuar el expediente y terminarlo forzosamente (mismo Final 5).

Las respuestas suelen constar de un mensaje de correo electrónico, al que se anexa uno o varios ficheros en Excel. Si son muchos se juntan todos en un ZIP. Cuando la explicación de la respuesta requiere un texto más largo, al mensaje de correo electrónico también se puede anexar un fichero Word, rtf o pdf, como se ha dicho antes. Igualmente, las consultas generadas por un cargo importante dirigidas al Director del Idescat se contestan adjuntando el pdf de una carta firmada por éste.

\section{Costes y beneficios}

Como se dijo, no hubo que pagar el coste base del programa ${ }^{3}$, porque ya lo había comprado la $\mathrm{Ge}$ neralitat (no sé cuánto es, pero seguramente no es mucho, pues lo la-

Dispersión de los tiempos de respuesta

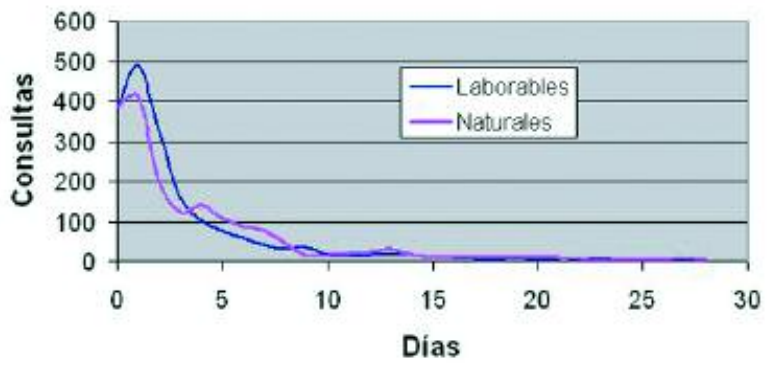

Figura 3. Tiempos de respuesta

borioso es su adaptación a la medida de las necesidades de cada institución).

El importe inicial fue de 39.140 euros, IVA incluido. Dada la complejidad que adquirió el sistema mientras se iba implementando por parte de un informático de STE trabajando en los locales del Idescat -lo que le permitía consultar y mantener reuniones continuadas-, se llegó a un acuerdo con STE para abonar 13.932 euros más (por ejemplo para hacer el formulario y su base de datos). En 2005 se abonaron 9.360 euros para realizar varias modificaciones y hacer el análisis funcional de la versión 2 (básicamente una simplificación de la 1). Finalmente en 2006 la consultora ha facturado 8.938 euros para la implementación de dicha nueva versión.

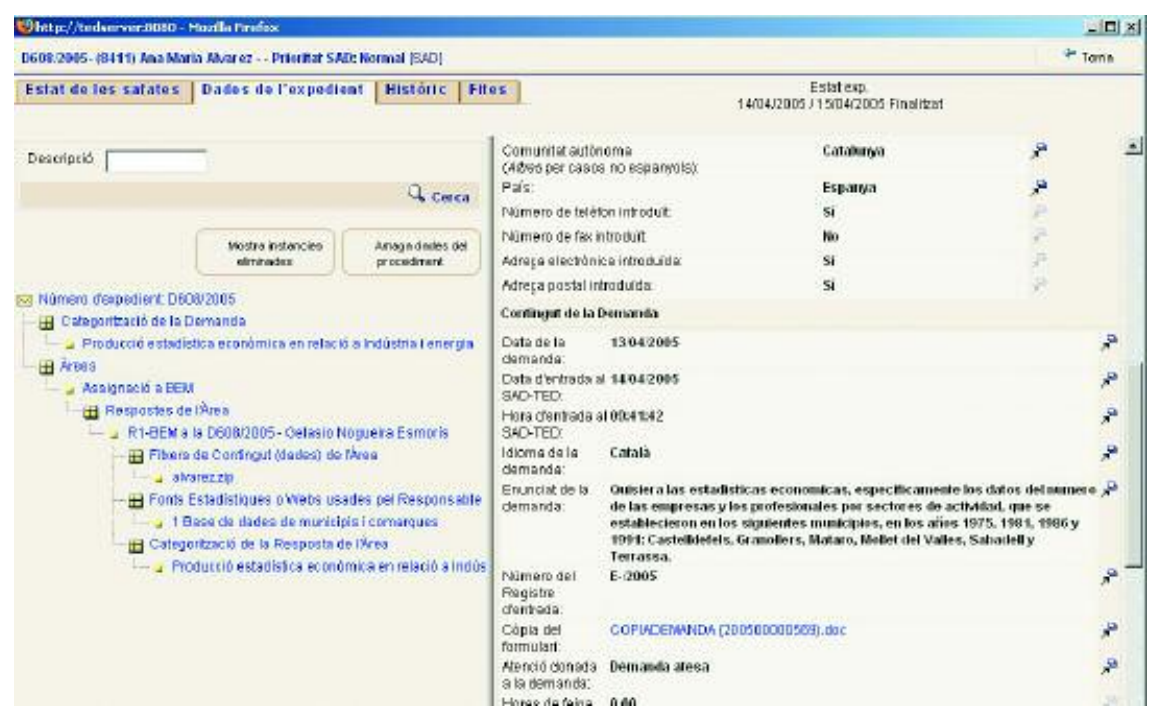

Figura 4. Seguimiento de un expediente, en este caso uno finalizado. Haciendo click en cualquier punto del árbol del expediente (a la izquierda) se puede acceder (a la derecha) a cada una de las etapas: pregunta del solicitante -etapa abierta para esta captura de pantalla-, comentarios internos, texto enviado al solicitante, abrir los ficheros de respuesta (alvarez.zip), fuentes estadísticas empleadas, tema, etc.
A esto hay que añadir un PC dedicado y una licencia de la base de datos Oracle.

Los beneficios intangibles no se han cuantificado: ahorro de tiempo de los técnicos estadísticos más cualificados, a los cuales les llegan menos consultas; ahorro en tiempo total de tramitación; mayor rapidez para los ciudadanos...

\section{La experiencia Idescat: ventajas e inconvenientes}

\section{Ventajas}

-El tiempo de respuesta a las demandas ha bajado drásticamente, con el 19\% de ellas contestadas en el mismo día (menos de 8 horas), y otro $19,8 \%$ en el día siguiente (figura 3).

- Se ha dado un importante paso hacia la homogeneización de procedimientos.

- Se han ahorrado muchas horas de técnico estadístico de producción al poder filtrar las consultas y hacerles llegar sólo aquellas más complejas que sólo pueden elaborar ellos.

-Fácil corrección de errores y retrocesión de expedientes.

- Cuando un ciudadano llama preguntando por la situación de su consulta cualquier persona del Idescat puede hacer un seguimiento del expediente (aunque para esto normalmente se pasa la llamada al $S A D$ ) (figura 4). 


\section{baratz}

\section{En primera línea en}

Sistemas de Información y Gestión del Conocimiento
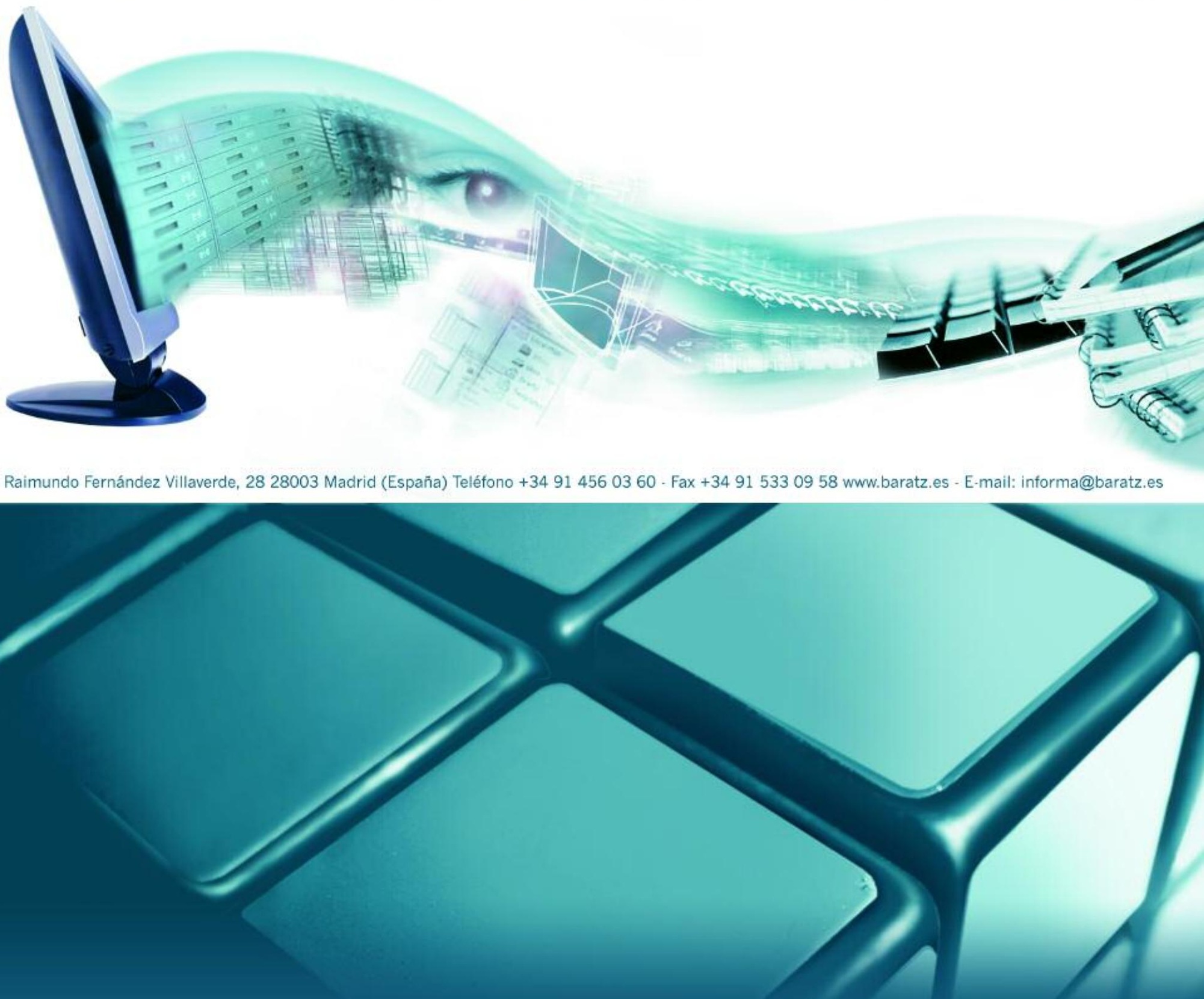

(2) Soluciones para bibliotecas: Absys, absys NET, Absys

(2) Gestión documental y del conocimiento: BKM, Baratz Windows, BRSCGI

(9) Soluciones para archivos: Albalá

(2) Servicios de Catalogación Retrospectiva

(9) Edición en CD-Rom

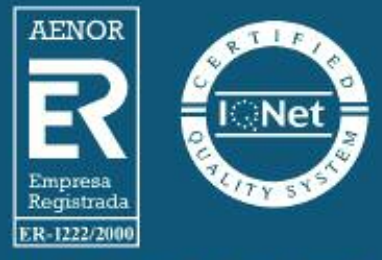


-En casos de enfermedad o época de vacaciones es fácil buscar técnicos sustitutos. El administrador del sistema puede hacer compartir bandejas aunque sean de personas con perfiles diferentes, y entonces los expedientes pueden ser tramitados indistintamente por cualquiera de ellas (mientras el trámite no se haya iniciado). Por supuesto, también existe la posibilidad de compartir contraseña de acceso desde cualquier PC. Si el técnico idóneo no está disponible consideramos que antes que no contestar siempre es mejor dar una respuesta o explicación amable, aunque sólo sea parcial u orientativa.

-El informe con las estadísticas del $S A D-T E D$ obtenidas a final de año (cantidad de consultas atendidas y tiempos de respuesta, total, por unidades y por técnicos) causaron impacto y fueron muy saludables para la posterior buena marcha del sistema en los meses siguientes. Algunas unidades se están esforzando en hacer aflorar todos los trabajos que antes se realizaban sin que quedara constancia.

—Evidentemente, todas las otras estadísticas que proporciona $S A D-T E D$ son muy interesantes: desde qué países se pide información, desde qué comarcas, sobre qué temas, instituciones oficiales, empresas, grado de agregación (sección censal, municipio, comarca, provincia...)

- SAD-TED ha sido la primera herramienta corporativa usada en el Idescat, contribuyendo al mantenimiento de su imagen y misión comunes entre el personal.

-Todos los técnicos y responsables del Instituto pueden ver todos los expedientes iniciados y finalizados, de forma transparente.

\section{Inconvenientes}

El único inconveniente está ya superado. Fue el esfuerzo económico y de tiempo dedicado a adecuar la aplicación a las necesidades reales de la organización. El paso del sistema manual anterior a un sistema realmente eficaz basado en el software TED costó un poco, pues como durante el diseño era difícil imaginar cuál sería el funcionamiento real se produjeron algunas de las situaciones clásicas, casi inevitables, en la implementación de proyectos, que ya se han comentado al principio. Se ha hablado de la complejidad innecesaria, pero por otra parte ¿cómo saber exactamente a priori lo que hacía falta y lo que sobraba?

Durante los primeros meses de funcionamiento las 2 personas a cargo del $S A D$ tuvieron que atender muchas dudas y problemas. STE también tuvo que subsanar bastantes bugs del programa, para lo cual mostró total disponibilidad y rapidez.

\section{Conclusiones}

¿Caro, barato, bueno, malo, recomendable...? ¡La difícil evaluación de los beneficios intangibles!

No se instala un servicio así ni cada día ni cada año, y no sé qué hubiera pasado si se hubiera contratado lo mismo a otra consultora y en otra época. Sólo puedo decir que al Idescat le ha ido bien, y que STE es una gran empresa con muchísima experiencia desarrollando aplicaciones en todo tipo de instituciones, en especial en administraciones públicas.

A pesar de que el número de peticiones recibidas en el servicio no es muy alto (unas 1.650 en 2005 , 1.900 previstas en 2006), creemos que ha valido claramente la pena instalar el $S A D-T E D$ para poderlas tramitar a plena satisfacción de los usuarios y sobre todo con el mínimo de recursos por parte del Instituto. La necesidad de este servicio se había presentado espontáneamente hace años, y si una vez asumido se decide que hay que ofre-

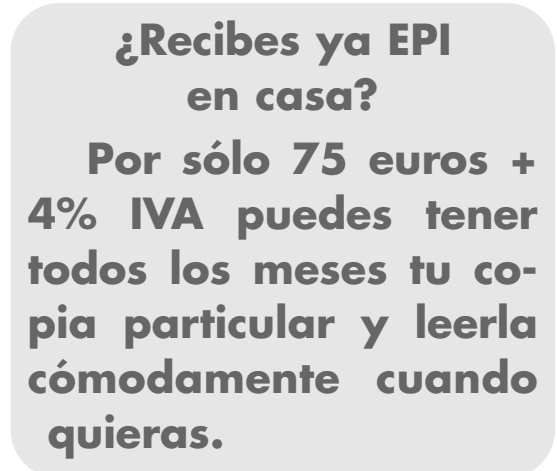

cerlo, hay que hacerlo bien. Bastantes consultas proceden de particulares y estudiantes (38\%) -la mayoría de las cuales vía $S A D-T E D$ son dirigidos a la Biblioteca-, pero el resto son técnicos de empresas, universidades, centros de investigación, administración pública, periodistas..., a los cuales el Instituto facilita su labor investigadora, de estudio y/o de divulgación. El Servicio no es multitudinario, pero incide en una capa bastante importante de la sociedad a la que no sólo es necesario servir con eficacia, sino que también conviene proyectar una buena imagen del Idescat.

\section{Notas}

1. STE Consulting es una empresa del grupo Altran

http://www.ste.es

http://www.altran.net

2. A efectos funcionales las «unidades» del $S A D$ no se corresponden con las «áreas» oficiales en las que está dividido el Instituto. Una de las ventajas de $T E D$ es la gran facilidad para modificar el organigrama interno del sistema, así como las asignaciones y perfiles de los distintos usuarios (director, subdirector, jefe de unidad, administrativo de unidad, técnico, administrador del sistema, administrador del $S A D$, etc.).

3. Es interesante saber que el software básico procede de la participación de $S T E$ en un proyecto subvencionado por la Comisión Europea, cosa que detectamos cuando en las primeras reuniones los técnicos hablaban de «librables» (deliverables), el término que usa la $C E$ para los diferentes documentos que se van produciendo a medida que se lleva a cabo un proyecto.

Tomàs Baiget, Institut d'Estadística de Catalunya, Via Laietana, 58. 08003 Barcelona.

Tel.: +34-934 120 088;

fax: 934123145

http://www.idescat.net

baiget@idescat.net

baiget@sarenet.es 